\title{
Entwicklung und Erprobung eines Kurzfragebogens zur Beurteilung von Trainer/innenkompetenzen in beruflichen Weiterbildungsmaßnahmen
}

\author{
A. Grohmann' - E.-M. Schulte' ${ }^{1}$ (D) S. Kauffeld ${ }^{1}$ \\ Angenommen: 14. Januar 2021 / Online publiziert: 28. Januar 2021 \\ (c) Der/die Autor(en) 2021
}

\section{Zusammenfassung}

In diesem Beitrag der Zeitschrift Gruppe. Interaktion. Organisation. (GIO) wird ein mehrdimensionaler und zeitlich effizienter Kurzfragebogen zur Beurteilung von Trainer/innenkompetenzen in beruflichen Weiterbildungsmaßnahmen vorgestellt und in drei Studien mit einer Gesamtstichprobe von $N=872$ Berufstätigen psychometrisch überprüft. Konfirmatorische Faktorenanalysen bestätigen die postulierte mehrdimensionale Faktorenstruktur (Methoden-, Fach-, Sozial- und Selbstkompetenz). Erste Hinweise auf die Kriteriumsvalidität des Fragebogens werden durch Analysen des Zusammenhangs mit Erfolgsmaßen (Zufriedenheit mit dem/der Trainer/in und mit der Weiterbildungsmaßnahme, wahrgenommener Transfer in den Arbeitsalltag) ermittelt. Theoretische und praktische Implikationen für den Einsatz in Unternehmen werden aufgezeigt.

Schlüsselwörter Fragebogenentwicklung · Trainer/innenkompetenzen · Weiterbildungserfolg · Zufriedenheit · Transfer

\section{Development and validation of a timeefficient questionnaire for assessing trainer competences in professional training programs}

\begin{abstract}
The present article in the journal Gruppe. Interaktion. Organisation. (GIO) aims at developing a multi-dimensional and time-efficient questionnaire for assessing trainer competences in professional training programs. Overall, three studies with a sample of $N=872$ employees were used to investigate the psychometric properties of the questionnaire. Confirmatory factor analyses underscore the importance of distinguishing different trainer competences (method, professional, social, and self-competence). Moreover, regression analyses with measures of training success (satisfaction with trainer and training, perceived transfer to practice) point to the questionnaire's criterion-related validity. Theoretical and practical implications for using the questionnaire in organizations are outlined.
\end{abstract}

Keywords Questionnaire development $\cdot$ Trainer competences $\cdot$ Training success $\cdot$ Satisfaction $\cdot$ Training transfer

Dipl-Psych. A. Grohmann

annagrohmann@aol.com

Dr. E.-M. Schulte

e.schulte@tu-braunschweig.de

Prof. Dr. S. Kauffeld

s.kauffeld@tu-braunschweig.de

1 Lehrstuhl für Arbeits-, Organisations- und

Sozialpsychologie, Technische Universität Braunschweig,

Spielmannstr. 19, 38106 Braunschweig, Deutschland
Für die Praxis und Forschung ist die Identifikation von förderlichen Einflussfaktoren auf den Weiterbildungserfolg bedeutsam, um diesen zu maximieren (z.B. Baldwin et al. 2017; Kauffeld 2016). Ein Erfolgsfaktor von Weiterbildungsmaßnahmen selbst ist der/die Durchführende der Maßnahme (z. B. Bell et al. 2011; Nor et al. 2017; Wißhak et al. 2020), der/die im Folgenden als Trainer/in bezeichnet wird. So zeigt sich bspw. für die Zufriedenheit mit dem/der Trainer/in ein substantieller Zusammenhang mit der Gesamtzufriedenheit mit der Weiterbildungsmaßnahme (Hochholdinger und Keller 2015). Die Bedeutsamkeit des/der Trainers/Trainerin sowie dessen/deren Kompeten- 
zen werden in der Forschung zunehmend betont (Bell et al. 2011; Gauld und Miller 2004; Harris et al. 2014; Nor et al. 2017; Wißhak et al. 2020). Dabei kommt der differenzierten Kompetenzerfassung eine besondere Bedeutung zu (Kauffeld und Paulsen 2018; für einen Überblick zu Kompetenzen von Trainer/innen im Rahmen beruflicher Weiterbildungen siehe Bonnes et al. 2019; Sgier und Lattke 2012), da so bspw. umfassende Ansatzpunkte zur Weiterentwicklung identifiziert werden können. Gleichzeitig sind Fragebögen zur differenzierten Einschätzung von Trainer/innenmerkmalen im Rahmen beruflicher Weiterbildung, die den Anforderungen der Praxis genügen, rar (Bell et al. 2011; Donovan und Darcy 2011; Kauffeld 2016). Bisherige ökonomische Fragebögen bzw. Skalen geben in der Regel nur einen Gesamtwert für die Trainer/ inneneinschätzung an (z.B. Morgan und Casper 2000), der keine Einschätzung erlaubt, ob ein spezifischer Aspekt (z.B. die Methodenkompetenz des/der Trainers/Trainerin) relevante Einflüsse auf den Maßnahmenerfolg zeigt oder nicht. Im vorliegenden Artikel soll daher ein vielseitig einsetzbarer Kurzfragebogen zur Erfassung von Trainer/ innenkompetenzen in beruflichen Weiterbildungsmaßnahmen entwickelt werden, der eine zeiteffiziente Evaluation in der Praxis (Kauffeld 2016) ermöglicht, aber trotzdem eine differenziertere Analyse von Trainer/innenmerkmalen (Bell et al. 2011) erlaubt, da explizit zwischen unterschiedlichen Kompetenzdimensionen unterschieden wird.

Im Folgenden stellen wir zunächst die Relevanz des gewählten Rahmenmodells, welches zwischen Methoden-, Sozial-, Fach- und Selbstkompetenz differenziert, vor. Anschließend geben wir einen Überblick über bestehende Kompetenzprofile und -kataloge der Trainer/innenforschung sowie vorhandene Fragebögen zur Messung einzelner Facetten der Trainer/innenkompetenz und leiten daraus den Bedarf eines neuen Kurzfragebogens ab. In allen Abschnitten liegt der Fokus auf der bisherigen Forschung zu Trainer/innen. Ergänzend werden Studien aus dem Kontext von Lehrkräften angeführt, da hinsichtlich des Lehrens und Instruierens zentrale Aufgaben vergleichbar sind (Bonnes et al. 2019) und so Erkenntnisse auch aus diesem Bereich als Grundlage der aktuellen Studie genutzt werden können.

\section{Rahmenmodell zur Erfassung von Trainer/ innenkompetenzen}

Um die Kompetenzen von Trainern/Trainerinnen im Rahmen beruflicher Weiterbildungsmaßnahmen $\mathrm{zu}$ erfassen, wurden verschiedene Rahmenmodelle vorgeschlagen (für den Bereich der Weiterbildung z.B. Garrick und McDonald 1992; für den angrenzenden Bereich der Ausbildung, z.B. Oser et al. 2009). Ein zentraler Aspekt der unterschiedlichen Kompetenzdefinitionen bezieht sich darauf, dass Kompetenzen in spezifischen Situationen in Form von Handlungen zum Ausdruck gebracht werden (Graf 2011; Sonntag und Schäfer-Rauser 1993; Wick 2009). Zahlreiche Einzelstudien belegen zwar die Relevanz unterschiedlicher Kompetenzfacetten (Bonnes et al. 2019), aber ein einheitliches Verständnis von den zentralen Kompetenzen fehlt bisher (Gauld und Miller 2004) ebenso wie ein Rahmenmodell zur Integration der heterogenen Forschungslandschaft (Bonnes et al. 2019).

Ein in anderen Kontexten weit verbreitetes Rahmenmodell zur Erfassung von Kompetenzen unterscheidet zwischen Methoden-, Fach-, Sozial- und personale bzw. Selbstkompetenz (z.B. Erpenbeck und von Rosenstiel 2007; Kauffeld 2006; Kauffeld und Paulsen 2018; Sonntag und Schaper 2006). Empirische Belege für die Unterscheidung dieser vier Kompetenzdimensionen finden sich bspw. bei Kauffeld und Henschel (2010), die Hinweise auf die zugrundeliegende vierfaktorielle Struktur im Kompetenz-Reflexions-Inventar (KRI), einem Fragebogen zur Selbsteinschätzung von Kompetenzen, ermittelt haben. Methodenkompetenz ist definiert, als ein breites Methodenrepertoire des/der Trainers/Trainerin, welches situationsübergreifend und flexibel eingesetzt werden kann (Burke und Hutchins 2008; Hughes et al. 1992; Kauffeld und Paulsen 2018). Trainer/innen mit einer hohen Methodenkompetenz gelingt es demnach Transferelemente zu integrieren (Schwaab 2002), den Wissensstand der Teilnehmenden einzuschätzen (Garrick und McDonald 1992) und Trainings interaktiv zu gestalten (Mamaqi et al. 2011). Fachkompetenz umfasst neben dem relevanten Wissen des jeweiligen Themas auch die Berufserfahrung sowie die Fähigkeit organisationales Wissen sinnorientiert einzuordnen, Probleme $\mathrm{zu}$ identifizieren und Lösungen $\mathrm{zu}$ generieren (Burke und Hutchins 2008; Kauffeld und Paulsen 2018). Die Sozialkompetenz umfasst die Fähigkeiten, ein positives Lernklima zu fördern (Harris et al. 2000), kommunikatives Geschick (Olson 1994; Schwaab 2002) sowie die Aktivierung und das Engagement der Teilnehmenden zu fördern (Arghode und Wang 2016; Graf 2011; Schwaab 2002). Die Selbstkompetenz beschreibt die Überzeugungskraft eines/ einer Trainers/Trainerin und dessen/deren Umgang mit unerwarteten Situationen (Schwaab 2002).

\section{Inventare zur Erfassung von Trainer/ innenkompetenzen}

Durch die Kombination von qualitativen und quantitativen Verfahren wurden verschiedene Kompetenzprofile und -kataloge entwickelt, welche die Kompetenzeinschätzung von Trainer/innen im Rahmen beruflicher Weiterbildungsmaßnahmen ermöglichen (für einen Überblick, siehe Bonnes et al. 2019 sowie Sgier und Lattke 2012). So werden im 
Rahmen des Projekts QF2TEACH ausgehend vom Europäischen Qualifikationsrahmen neun Kernkompetenzen sowie eine Vielzahl von Teilkompetenzen unterschieden und in der Schweizer Version den beiden Bereichen (1) Methodenund Fachkompetenz sowie (2) Sozial- und Selbstkompetenz zugeordnet (Sgier 2012). Wißhak und Hochholdinger (2018) leiten basierend auf einer umfassenden Recherche in einer aktuellen Studie zu relevantem Wissen und Fähigkeiten von Trainern/Trainerinnen insgesamt 41 relevante Facetten ab, welche sie in 10 Kategorien (z. B. Planung, methodische Implementierung des Trainings, Fachwissen) gruppieren. Auch wenn eine Beurteilung der Wichtigkeit dieser Facetten durch Trainer/innen, Teilnehmende und HR Verantwortliche wichtige Einblicke in die Praxisrelevanz von Wissen und Fähigkeiten gibt, steht eine empirische Überprüfung der theoretisch abgeleiteten Kategorien aus. Bonnes und Kollegen (2019) fassen die aktuelle Literatur zu Trainer/innenkompetenzen zu sieben Kompetenzaspekten zusammen, wie bspw. das Wissen zur Planung und Strukturierung von Trainings, Wissen des und Erfahrung im jeweiligen Fachgebiet, die Fähigkeit Gruppen zu managen sowie persönliches Auftreten und Enthusiasmus des/der Trainers/in. Somit finden sich - neben anderen Aspekten - auch bei Bonnes et al. (2019) die vier Kompetenzdimensionen Methoden-, Fach-, Sozial- und Selbstkompetenz inhaltlich wieder. Ein entsprechender Fragebogen hierzu liegt noch nicht vor. Ein weiteres umfassendes Inventar zu Kompetenzen von Lehrenden in der Erwachsenen- und Weiterbildung wurde im Rahmen des Projekts GRETA (Bosche et al. 2018) entwickelt. Es umfasst vier Kompetenzaspekte (berufspraktisches Wissen und Können, fach- und feldspezifisches Wissen, professionelle Selbststeuerung und professionelle Werthaltungen und Überzeugungen) sowie 12 Kompetenzbereiche und 25 Kompetenzfacetten, zu denen noch fachspezifische Facetten hinzukommen. Der Fokus dieses Inventars liegt jedoch darauf Trainer/innenkompetenzen zu bilanzieren und validieren zu lassen: Trainer/innen bearbeiten das Portfolio mit allen Kompetenzfacetten selbstständig (Selbstreflexion) und reichen dies dann mit dem Ziel einer Zertifizierung zur Begutachtung ein. Es ist hingegen aufgrund der Ziele als auch der Länge nicht geeignet, um eine Fremdeinschätzung der Trainer/innenkompetenzen durch Trainingsteilnehmende vorzunehmen.

Neben diesen umfassenden Ansätzen, die zur Identifikation von relevanten Kompetenzfacetten dienen, aber keine wissenschaftlich überprüften Instrumente zur Fremdeinschätzung beinhalten, liegen für einzelne Trainer/innenkompetenzen zudem Fragebögen, Skalen bzw. Items vor: Die Sozialkompetenz kann bspw. mit einem 20-Item-Fragebogen von Graf (2011) erfasst werden. Zudem beinhalten weitere Fragebögen einzelne Items zur Sozialkompetenz (z.B. Morgan und Casper 2000; Stoughton et al. 2011). Die Methodenkompetenz lässt sich bspw. mit einer 4-Item-
Skala von Holton et al. (2000) erfassen: Sie fokussiert auf das Transfer-Design, also das Ausmaß, in dem die vom Trainer oder der Trainerin verwendeten Übungen relevant für den Arbeitsalltag bzw. transferförderlich sind. Weitere Items finden sich auch hier bei Morgan und Casper (2000) sowie bei Stoughton et al. (2011). Zur Selbstkompetenz finden sich bspw. Items im LEADS Fragebogen, der unter anderem die Facette Enthusiasmus beinhaltet (RussEft et al. 2010) sowie einzelne Items in der Skala von Stoughton et al. (2011). Fachkompetenz wird bspw. mit dem Instrument zum pädagogisch-psychologischen Wissen sowohl für Lehrkräfte an Schulen als auch im Bereich der Erwachsenbildung mittels Text und videobasierten Aufgaben erfasst (Marx et al. 2017) und ist ebenfalls Bestandteil des LEADS Fragebogens (Russ-Eft et al. 2010).

Trotz der umfassenden qualitativen Studien zu relevanten Facetten der Trainer/innenkompetenzen sowie der quantitativen Analysen zu einzelnen Kompetenzfacetten, steht die Entwicklung eines psychometrisch überprüften, wissenschaftlich fundierten sowie in einem breiten Spektrum beruflicher Weiterbildungsmaßnahmen einsetzbaren Fragebogens, der die vier Kompetenzdimensionen eines/einer Trainers/Trainerin (Methoden-, Fach-, Sozial- und Selbstkompetenz) explizit unterscheidet, aus. Da zur Bewältigung der Herausforderungen der heutigen Arbeitswelt zudem zeitsparende Instrumente zur Evaluation von Weiterbildungsmaßnahmen essenziell sind (Kauffeld 2016), ist es Ziel dieser Studie einen ökonomischen Kurzfragebogen zur Beurteilung von Trainer/innenkompetenzen in beruflichen Weiterbildungsmaßnahmen zu entwickeln und zu überprüfen. Basierend auf dem bestehenden Rahmenmodell zur Einteilung von Kompetenzen in Methoden-, Fach-, Sozial- und Selbstkompetenz (z. B. Erpenbeck und von Rosenstiel 2007; Kauffeld 2006; Kauffeld und Paulsen 2018; Sonntag und Schaper 2006) sowie der identifizierten relevanten Kompetenzfacetten von Trainer/innen (Bonnes et al. 2019; Mamaqi et al. 2011; Wißhak und Hochholdinger 2018), nehmen wir an, dass - im Sinne eines ökonomischen Fragebogens die zentralen Aspekte der Trainer/innenkompetenz mittels dieser vier Kompetenzfacetten erfasst werden können.

Hypothese 1 Der Kurzfragebogen weist eine vierfaktorielle Struktur (Methoden-, Fach-, Sozial- und Selbstkompetenz) auf.

\section{Zusammenhänge zwischen Trainer/innenkompetenzen und Weiterbildungserfolg}

Bisherige Forschung zu Trainer/innenkompetenzen zeigt positive Effekte der Trainerin bzw. des Trainers auf relevante Erfolgsmaße (Hochholdinger und Keller 2015; Morgan 
und Casper 2000; Russ-Eft et al. 2010). Um erste Hinweise zur Validität des neu entwickelten Kurzfragebogen zu erhalten, wird daher entsprechend theoretischer Annahmen (Kirkpatrick und Kirkpatrick 2006; Wang und Wilcox 2006) der Zusammenhang der vier Kompetenzbereiche mit sowohl kurzfristigen (Zufriedenheit mit dem/der Trainer/in sowie Zufriedenheit mit der Weiterbildungsmaßnahme insgesamt) als auch langfristigen (Transfer des Gelernten in den Arbeitsalltag) Trainingserfolgsmaßen untersucht.

Der Zusammenhang zwischen einzelnen Trainer/innenmerkmalen und der Gesamtzufriedenheit mit dem/der Trainer/in wurde im Hochschulbereich empirisch belegt: So weisen Merkmale von Lehrenden (Umgang mit den Studierenden, Interessantheit und Relevanz sowie Planung und Darstellung), substantielle Zusammenhänge mit der Gesamtzufriedenheit mit dem/der Lehrenden in Seminaren auf (Staufenbiel 2000). Für den Bereich der beruflichen Weiterbildung werden daher auch für alle vier Trainer/ innenkompetenzbereiche Zusammenhänge mit der Gesamtzufriedenheit erwartet.

Hypothese 2 Die Methoden- (H2a), Fach- (H2b), Sozial(H2c) und Selbstkompetenz (H2d) eines/einer Trainers/ Trainerin hängen positiv mit der Gesamtzufriedenheit mit dem/der Trainer/in zusammen.

Empirische Studien zeigen einen Zusammenhang zwischen der Bewertung des/der Trainers/Trainerin und den Reaktionen der Teilnehmenden auf die Weiterbildungsmaßnahme, bspw. der Gesamtzufriedenheit mit der beruflichen Weiterbildungsmaßnahme (Hochholdinger und Keller 2015; Morgan und Casper 2000). Dieses Bild wird in Dozierendenbewertungen im Hochschulbereich bestätigt: Bei Seminaren zeigen sich ebenfalls für die Skalen, die sich insbesondere auf Merkmale von Lehrenden beziehen, substantielle Zusammenhänge mit der Gesamtzufriedenheit mit der Veranstaltung (Staufenbiel 2000). Zudem belegt eine Studie den zusätzlichen Effekt der Trainer/innenkompetenz zu motivationalen Aspekten der Teilnehmenden auf die Zufriedenheit Studierender mit dem Seminar (Harris et al. 2014).

Hypothese 3 Die Methoden- (H3a), Fach- (H3b), Sozial(H3c) und Selbstkompetenz (H3d) eines/einer Trainers/ Trainerin weisen einen positiven Zusammenhang mit der Gesamtzufriedenheit mit der Weiterbildungsmaßnahme auf.

Über die in der Praxis häufig untersuchten Reaktionen der Teilnehmenden hinaus (Blume et al. 2010; Sitzmann und Weinhardt 2019), sollten Unternehmen evaluieren, ob die Weiterbildungsinhalte tatsächlich in den Arbeitsalltag transferiert werden (Baldwin et al. 2017; Blume et al. 2010; Kauffeld 2016). Erste Hinweise auf einen möglichen transferförderlichen Einfluss von Aspekten, die der Methodenkompetenz des/der Trainers/Trainerin zugeordnet werden können, zeigt eine Studie von Lim (2000). Zudem finden sich weitere Hinweise zur Relevanz des/der Trainers/ Trainerin für den Transfer in Studien, die einzelne Aspekte untersuchen, welche maßgeblich von dem/der Trainer/in beeinflusst werden: So zeigt sich ein Zusammenhang des Transferdesigns und der Transferqualität, Transferquantität sowie der wahrgenommenen Anwendung in der Praxis (Selbst- und Fremdeinschätzung) vermittelt über die Transfermotivation (Grohmann et al. 2014). Die Ausdrucksfähigkeit des/der Trainers/Trainerin zeigt in einem experimentellen Setting zudem einen maßgeblichen Effekt auf die Problemlösefähigkeit der Teilnehmenden (Towler 2009). Daher nehmen wir an, dass auch eine hohe Ausprägung in den vier Kompetenzbereichen positiv mit dem wahrgenommenen Transfer der Teilnehmenden zusammenhängt.

Hypothese 4 Die Methoden- (H4a), Fach- (H4b), Sozial(H4c) und Selbstkompetenz (H4d) eines/einer Trainers/ Trainerin weisen einen positiven Zusammenhang mit dem wahrgenommenen Transfer der Weiterbildungsinhalte in den Arbeitsalltag auf.

\section{Methode}

Ziel der vorliegenden Studie ist somit die Entwicklung und Überprüfung eines Kurzfragebogens zur Erfassung von Trainer/innenkompetenzen. Der Fragebogen wurde dabei mittels einer Kombination des Prototypenansatzes mit der rationalen Methode entwickelt (Bühner 2011). Um eine umfassende Grundlage für die spätere Itemgenerierung zu entwickeln sowie eine ganzheitliche Sichtweise auf Trainer/innenkompetenzen zu ermöglichen, wurden im ersten Schritt insgesamt 26 Interviews mit Experten und Expertinnen unter Verwendung des Prototypenansatzes (Bühner 2011) durchgeführt. An den Interviews beteiligten sich insgesamt 26 Personen, die im Durchschnitt 9,5 Jahre (Minimum $=1,5$ Jahre; Maximum $=30$ Jahre) Erfahrungen als Trainer/in gesammelt haben. Es nahmen 16 weibliche und 10 männliche Trainer/innen an den Interviews teil. Die Trainer/innen wurden in halbstrukturierten Interviews unter anderem nach den zentralen Merkmalen eines/einer erfolgreichen Trainers/Trainerin gefragt, um so im Sinne des Prototypenansatzes (vgl. Bühner 2011) die typischen Eigenschaften eines/einer Trainers/Trainerin zu identifizieren. Zudem wurden die für den Transfererfolg relevanten Verhaltensweisen eines/einer Trainers/Trainerin erfasst. Darüber hinaus wurden ausgewählte verhaltensnahe Fragen auf Basis der Critical Incident Technique (Flanagan 1954) gestellt, um erfolgskritische Verhaltensweisen differenziert zu erfassen (z.B. wurde eine Situation erfragt, in der sich das Verhalten des/der Trainers/Trainerin besonders positiv auf die Trainingssituation ausgewirkt hat). Der ratio- 
nalen Methode (Bühner 2011) folgend wurde anschließend ein Kompetenzmodell, das die Fach-, Methoden-, Sozialund Selbstkompetenz (Erpenbeck und von Rosenstiel 2007; Kauffeld 2006; Kauffeld und Paulsen 2018; Sonntag und Schaper 2006) eines/einer Trainers/Trainerin unterscheidet als Rahmenmodell für die inhaltsanalytische Auswertung (Mayring, 2010) der Interviews verwendet. Die Interviews wurden in Sinneinheiten unterteilt (Kauffeld 2006; Mayring 2010) und den vier Kompetenzdimensionen zugeordnet. Auf Basis der theoretisch postulierten vier Kompetenzdimensionen und den inhaltsanalytisch identifizierten Teilaspekten wurde anschließend ein umfassender Itempool generiert. Zur Reduktion und Überprüfung der Items wurden anschließend 3 Studien durchgeführt: In Studie 1 wurde auf Basis des Itempools (65 Items) eine Kurzform mit 16 Items mittels konfirmatorischer Faktorenanalysen identifiziert. In Studie 2 wurde die Kurzform kreuzvalidiert. Studie 3 diente der Analyse der Kriteriumsvalidität. Die Stichproben, das methodische Vorgehen sowie die Ergebnisse der drei Studien werden im Folgenden dargestellt.

\subsection{Stichproben}

Zur Prüfung der faktoriellen Struktur (Hypothese 1) sowie der Kriteriumsvalidität (Hypothese 2, 3 und 4) wurden drei Gelegenheitsstichproben mittels retrospektiver Online-Befragung erhoben. Die Teilnehmenden sollten die letzte berufliche Weiterbildungsmaßnahme, an der sie teilgenommen haben, einschätzen. Im vorliegenden Artikel wird dabei eine breitere Definition beruflicher Weiterbildung verwendet, die formalisierte (z.B. Trainings in Organisationen), arbeitsnahe (z.B. Workshop zu spezifischen beruflichen Inhalten) und selbst organisierte Formen (z.B. Abendlehrgang) unterscheidet (Beicht et al. 2006, S. 10f.). Neben der beruflichen Weiterbildungsmaßnahme sollte ebenfalls der/die Trainer/in der beruflichen Weiterbildungsmaßnahme bewertet werden. In allen drei Studien wurden Weiterbildungsmaßnahmen von unterschiedlichem Umfang und aus unterschiedlichen Bereichen eingeschätzt (z.B. fachliche Maßnahmen, etwa Buchhaltungsschulungen sowie überfachliche Maßnahmen, z.B. Trainings zum Thema Konfliktmanagement). Um die Datenqualität der Befragungen sicher zu stellen, wurden nur Berufstätige berücksichtigt, die die Fragen nach eigenen Angaben ernsthaft bearbeitet haben (für ein vergleichbares Vorgehen, siehe z.B. Grohmann und Kauffeld 2013). Zudem wurden nur berufliche Weiterbildungsmaßnahmen für die späteren Analysen verwendet, die maximal zwei Jahre zurück liegen, um mögliche Erinnerungseffekte zu vermeiden (Blau et al. 2012; Grohmann und Kauffeld 2013). Im Folgenden werden jeweils die finalen Stichproben beschrieben.

Stichprobe 1 umfasste $N=294$ Teilnehmende mit einem durchschnittlichen Alter von $M=33,75$ Jahre $(S D=10,81$;
$1 \%$ keine Angabe). Die Geschlechterverteilung war wie folgt: $43 \%=$ männlich, $56 \%$ weiblich (1\% keine Angabe). Die berufliche Weiterbildungsmaßnahme lag durchschnittlich 7,45 Monate zurück $(S D=6,42)$ und umfasste im Schnitt 116,36h $(S D=342,87)$.

Stichprobe 2 setzte sich aus $N=453$ Teilnehmenden zusammen, die im Durchschnitt $M=37,68$ Jahre alt waren $(S D=10,51 ; 1 \%$ keine Angabe). An der Studie nahmen $44 \%$ männliche, $56 \%$ weibliche Personen teil. Die berufliche Weiterbildungsmaßnahme lag im Schnitt 7,17 Monate zurück $(S D=6,33)$ und hatte eine Dauer von durchschnittlich 97,42 h $(S D=480,93 ; 1 \%=$ keine Angabe $)$.

Stichprobe 3 bestand aus $N=125$ Teilnehmenden mit einem durchschnittlichen Alter von $M=36,58$ Jahre $(S D=11,71)$. Es nahmen $42 \%$ männliche und $58 \%$ weibliche Befragte teil. In diese Stichprobe wurden nur Teilnehmende aufgenommen, deren Teilnahme an der beruflichen Weiterbildungsmaßnahme mindestens einen Monat zurücklag, um einen Transfer des Gelernten in die berufliche Praxis zu ermöglichen (für ein vergleichbares Vorgehen, siehe Grohmann und Kauffeld 2013). Im Durchschnitt lag die Weiterbildungsmaßnahme 6,98 Monate zurück $(S D=6,19)$ und hatte einen durchschnittlichen Umfang von $66,38 \mathrm{~h}(S D=174,41)$.

\subsection{Erhebungsinstrumente}

In Studie 1 wurden die aus den Interviews abgeleiteten 65 Items erhoben. In Studie 2 und 3 wurde der auf Grundlage der Itemselektion reduzierte Fragebogen zur Erfassung von Trainer/innenkompetenzen erhoben (vgl. Tab. 1). Es wurde eine 11-stufige Antwortskala mit den Antwortoptionen $0 \%=$ trifft überhaupt nicht $z u$ (kodiert als 0) bis $100 \%=$ trifft völlig zu (kodiert als 10) verwendet (analog z.B. zu Grohmann und Kauffeld 2013).

Validierungsmaße: In Studie 3 wurde die Gesamtbewertung des/der Trainers/Trainerin mit dem Item ,Welche Note würden Sie dem Trainer/der Trainerin - als Leiter/in der Veranstaltung - geben?“ erfasst (Staufenbiel 2000; adaptiert an den Befragungskontext). Die Einschätzung der Gesamtzufriedenheit mit dem Lehrpersonal und der Veranstaltung mittels Schulnotenskalen ist eine weit verbreitete Evaluationsform im Bereich der pädagogischen Psychologie, speziell in der Hochschullehrevaluation (z. B. Staufenbiel 2000) sowie in der Praxis beruflicher Weiterbildungsmaßnahmen (z. B. Hölbling 2007). Als Antwortskala wurde eine Schulnotenskala von $1=$ sehr gut bis $6=$ ungenügend verwendet (Staufenbiel 2000). Um vergleichbare Ergebnisse $\mathrm{zu}$ erhalten, wurde mit der gleichen Antwortskala die Gesamtzufriedenheit mit der Weiterbildungsmaßnahme mit dem Item ,Welche Note würden Sie dem Training insgesamt geben?" erfasst (Staufenbiel 2000; adaptiert an den Befragungskontext). Zu Beginn der Befragung wurde in 
Tab. 1 Itemkennwerte (Mittelwerte, Standardabweichungen und korrigierte Trennschärfen) von Studie 1

\begin{tabular}{|c|c|c|c|c|c|}
\hline Item & Der Trainer/Die Trainerin ... & $N$ & $M$ & $S D$ & $r_{i t}{ }^{\mathrm{a}}$ \\
\hline MK1 & ... hat konsequent den roten Faden der Veranstaltung verdeutlicht & 294 & 7,50 & 2,65 & 0,69 \\
\hline MK2 & ... hat an das Vorwissen der Teilnehmenden angeknüpft & 292 & 7,18 & 2,87 & 0,66 \\
\hline MK3 & ... hat genau die richtigen Methoden angewendet, um die Trainingsinhalte zu vermitteln & 293 & 7,29 & 2,47 & 0,75 \\
\hline MK4 & $\begin{array}{l}\text {... hat eine sehr gute Verbindung zwischen den Trainingsinhalten und dem Arbeitsall- } \\
\text { tag hergestellt }\end{array}$ & 291 & 7,55 & 2,66 & 0,75 \\
\hline FK1 & ... brachte sein/ihr Praxiswissen ein & 291 & 8,55 & 2,38 & 0,86 \\
\hline FK2 & ... hat sehr viel Erfahrung mit den Trainingsinhalten & 291 & 8,84 & 2,04 & 0,85 \\
\hline FK3 & ... konnte auf alle fachlichen Fragen überzeugend antworten & 291 & 8,47 & 2,23 & 0,78 \\
\hline FK4 & ... kannte sich in dem Trainingsthema exzellent aus & 291 & 8,71 & 2,12 & 0,89 \\
\hline SoK1 & ... ging sehr individuell auf die Bedürfnisse der Teilnehmenden ein & 292 & 6,95 & 2,94 & 0,70 \\
\hline SoK2 & ... verwendete eine für die Teilnehmenden angemessene Wortwahl & 294 & 8,83 & 1,61 & 0,56 \\
\hline SoK3 & ... hat die Teilnehmenden für die Trainingsinhalte begeistert & 290 & 7,37 & 2,67 & 0,75 \\
\hline SoK4 & ... hat einen guten Zugang zu den Teilnehmenden gefunden & 292 & 7,87 & 2,37 & 0,85 \\
\hline SeK1 & ... Ist mit kritischen Situationen gelassen umgegangen & 290 & 7,76 & 2,52 & 0,75 \\
\hline $\mathrm{SeK} 2$ & ... konnte sich gut auf neue Situationen einstellen & 294 & 7,72 & 2,66 & 0,83 \\
\hline SeK3 & ... war sehr engagiert & 293 & 8,62 & 1,86 & 0,74 \\
\hline $\mathrm{SeK} 4$ & ... hatte Überzeugungskraft & 294 & 8,20 & 2,41 & 0,84 \\
\hline
\end{tabular}

Inhaltlich ähnliche Items zu ausgewählten Aspekten des/der Trainers/Trainerin und zu Trainer/innenkompetenzen finden sich z. B. bei Graf (2011), Holton et al. (2000), Morgan und Casper (2000), Schwaab (2002), Sgier und Lattke (2012), Staufenbiel (2000) sowie Stoughton et al. (2011)

aListenweiser Fallausschluss

der Instruktion deutlich gemacht, dass mit der Bezeichnung Training im Folgenden unterschiedliche Formen beruflicher Weiterbildungsmaßnahmen (bspw. Seminare) gemeint sind. Beide Items wurden vor den Analysen rekodiert. Zur Erfassung des Transfers in die Praxis wurde die Skala wahrgenommene Anwendung in der Praxis aus dem Questionnaire for Professional Training Evaluation (Grohmann und Kauffeld 2013) verwendet. Ein Beispielitem der insgesamt zwei Items umfassenden Skala lautet: „Es gelingt mir sehr gut, die erlernten Trainingsinhalte in meiner täglichen Arbeit anzuwenden" (Grohmann und Kauffeld 2013). Es wurde die gleiche 11-stufige Antwortskala wie bei den Items zur Erfassung von Trainer/innenkompetenzen eingesetzt. Die Reliabilität der Skala ist mit $\alpha=0,86$ als gut einzuschätzen.

\subsection{Analysen}

Um einen möglichst ökonomischen Fragebogen zu entwickeln, wurden gemäß Bühner (2011, S. 432) vier Items pro Kompetenzdimension angestrebt (siehe Tab. 1 für die konkreten Items). In Studie 1 wurden im ersten Schritt die Itemverteilungen, Itemschwierigkeiten und Trennschärfen betrachtet (Bühner 2011; Fisseni 2004) und darauf basierend vier Items von weiteren Analysen ausgeschlossen. Mittels konfirmatorischer Faktorenanalysen wurde der Fragebogen nun sukzessive gemäß Bühner (2011) auf Basis von methodischen (bspw. Modifikationsindizes) sowie inhaltlichen Kriterien (bspw. Formulierung von Iteminhalten) auf insgesamt 16 Items, d.h. vier Items pro Kompetenzdi- mension reduziert. Da mit 61 Items und einer Stichprobe von $N=294$ das Verhältnis von freien Parametern zur Anzahl an Versuchspersonen nicht den empfohlenen Mindestwert von fünf erreichte (Bentler und Chou 1987), wurde die Itemreduktion in Studie 1 einzeln pro Kompetenzdimension und nicht im gesamten vierfaktoriellen Modell vorgenommen. In Studie 2 sollte die reduzierte Fragebogenform an einer neuen Stichprobe kreuzvalidiert werden. Das Verhältnis von freien Parametern zur Anzahl an Versuchspersonen war dabei ausreichend für eine Prüfung des kompletten Modells mit 16 Items und vier latenten Kompetenzdimensionen (Bentler und Chou 1987). Die konfirmatorischen Faktorenanalysen wurden mit Mplus 6 unter Verwendung von Full Information Maximum Likelihood (FIML) und eines robusten Maximum Likelihood-Schätzers (MLR) durchgeführt (Muthén und Muthén 2010). Zur Prüfung der Modellgüte wurden gemäß Kline (2005, S. 134) RMSEA, CFI und SRMR sowie das Verhältnis von $\chi^{2}$ zur Anzahl an Freiheitsgraden $d f$ verwendet (Schweizer 2010). Die Überprüfung der Kriteriumsvalidität wurde in Studie 3 mittels Regressionsanalysen in SPSS mit der Methode „Einschluss“ bei gleichzeitiger Berücksichtigung aller Prädiktoren realisiert. 


\section{Ergebnisse}

\subsection{Faktorielle Struktur}

In Studie 1 wurde mittels konfirmatorischer Faktorenanalysen pro Kompetenzdimension (Methoden-, Fach-, Sozialund Selbstkompetenz) jeweils sukzessive ein finales einfaktorielles Modell mit vier Items identifiziert. Die Modelle erreichen für jede der vier Kompetenzdimensionen eine gute Modellpassung (siehe Tab. 2; einen Überblick über CutOff-Werte gibt Schweizer 2010). Die Itemkennwerte (Mittelwerte, Standardabweichungen und Trennschärfen) sind Tab. 1, die Reliabilitäten und Skaleninterkorrelationen sind Tab. 3 zu entnehmen.
Um die faktorielle Struktur der in Studie 1 identifizierten Fragebogenform in ihrer Gesamtheit zu prüfen, wurde eine Kreuzvalidierung des gesamten Modells, bestehend aus vier latenten Kompetenzdimensionen und vier Items pro Dimension, in Studie 2 vorgenommen. Zur Prüfung der Angemessenheit des vierfaktoriellen Modells wurden zudem theoretisch plausible konkurrierende ein- und zweifaktorielle Modelle getestet (siehe Tab. 2). Im einfaktoriellen Modell A laden alle 16 Items auf einen latenten Faktor. Hierbei gehen wir von der Annahme eines globalen Kompetenzfaktors aus (vgl. z.B. Morgan und Casper 2000). Im zweifaktoriellen Modell B laden die Methoden- und Fachkompetenzitems auf einem Faktor sowie die Sozial- und Selbstkompetenzitems auf dem zweiten Faktor (für eine ähnliche Unterscheidung, siehe Sonntag und Schaper 2006, S. $283 \mathrm{ff}$.).

Tab. 2 Ergebnisse der konfirmatorischen Faktorenanalysen der finalen Modelle in Studie 1 und Studie 2

\begin{tabular}{|c|c|c|c|c|c|c|c|c|}
\hline & $N$ & Itemanzahl & $\chi^{2}$ & $D f$ & $\chi^{2} / d f$ & RMSEA & CFI & SRMR \\
\hline \multicolumn{9}{|l|}{ Studie 1} \\
\hline Methodenkompetenz (MK) & 294 & 4 & 1,90 & 2 & 0,95 & 0,00 & 1,00 & 0,01 \\
\hline Fachkompetenz (FK) & 291 & 4 & 3,42 & 2 & 1,71 & 0,05 & 1,00 & 0,01 \\
\hline Sozialkompetenz (SoK) & 294 & 4 & 0,27 & 2 & 0,13 & 0,00 & 1,00 & 0,00 \\
\hline Selbstkompetenz (SeK) & 294 & 4 & 0,32 & 2 & 0,16 & 0,00 & 1,00 & 0,00 \\
\hline \multicolumn{9}{|l|}{ Studie 2} \\
\hline $\begin{array}{l}\text { Modell A: } 1 \text { latenter Faktor } \\
(\mathrm{MK}+\mathrm{FK}+\mathrm{SoK}+\mathrm{SeK})\end{array}$ & 453 & 16 & 563,52 & 104 & 5,42 & 0,10 & 0,86 & 0,05 \\
\hline $\begin{array}{l}\text { Modell B: } 2 \text { latente, interkorrelierte Faktoren } \\
(\mathrm{MK}+\mathrm{FK}, \mathrm{SoK}+\mathrm{SeK})\end{array}$ & 453 & 16 & 442,54 & 103 & 4,30 & 0,09 & 0,89 & 0,06 \\
\hline $\begin{array}{l}\text { Modell C: } 2 \text { latente, interkorrelierte Faktoren } \\
(\mathrm{FK}, \mathrm{MK}+\mathrm{SoK}+\mathrm{SeK})\end{array}$ & 453 & 16 & 296,29 & 103 & 2,88 & 0,06 & 0,94 & 0,04 \\
\hline $\begin{array}{l}\text { Modell D: } 4 \text { latente, interkorrelierte Faktoren } \\
\text { (MK, FK, SoK, SeK) }\end{array}$ & 453 & 16 & 205,06 & 98 & 2,09 & 0,05 & 0,97 & 0,04 \\
\hline
\end{tabular}

Tab. 3 Manifeste Produkt-Moment-Korrelationen der Skalen des Kurzfragebogens zur Erfassung von Trainer/innenkompetenzen in allen Studien

\begin{tabular}{|c|c|c|c|c|c|}
\hline Skalen & Anzahl Items & $\begin{array}{l}\text { (1) } \\
\text { MK }\end{array}$ & $\begin{array}{l}\text { (2) } \\
\text { FK }\end{array}$ & $\begin{array}{l}\text { (3) } \\
\text { SoK }\end{array}$ & $\begin{array}{l}\text { (4) } \\
\text { SeK }\end{array}$ \\
\hline \multicolumn{6}{|l|}{ Studie 1} \\
\hline (1) Methodenkompetenz & 4 & $(0,86)$ & - & - & - \\
\hline (2) Fachkompetenz & 4 & $0,72^{* *}$ & $(0,93)$ & - & - \\
\hline (3) Sozialkompetenz & 4 & $0,81^{* *}$ & $0,74^{* *}$ & $(0,85)$ & - \\
\hline (4) Selbstkompetenz & 4 & $0,80^{* *}$ & $0,77^{* *}$ & $0,86^{* *}$ & $(0,90)$ \\
\hline \multicolumn{6}{|l|}{ Studie 2} \\
\hline (1) Methodenkompetenz & 4 & $(0,79)$ & - & - & - \\
\hline (2) Fachkompetenz & 4 & $0,69^{* *}$ & $(0,92)$ & - & - \\
\hline (3) Sozialkompetenz & 4 & $0,79^{* *}$ & $0,71^{* *}$ & $(0,85)$ & - \\
\hline (4) Selbstkompetenz & 4 & $0,76^{* *}$ & $0,71^{* *}$ & $0,82^{* *}$ & $(0,92)$ \\
\hline \multicolumn{6}{|l|}{ Studie 3} \\
\hline (1) Methodenkompetenz & 4 & $(0,81)$ & - & - & - \\
\hline (2) Fachkompetenz & 4 & $0,62^{* *}$ & $(0,94)$ & - & - \\
\hline (3) Sozialkompetenz & 4 & $0,75^{* *}$ & $0,62^{* *}$ & $(0,85)$ & - \\
\hline (4) Selbstkompetenz & 4 & $0,74^{* *}$ & $0,70^{* *}$ & $0,83^{* *}$ & $(0,91)$ \\
\hline
\end{tabular}

Reliabilität (Cronbach's Alpha) sind jeweils in der Diagonalen angegeben ${ }^{*} p<0,05 ;{ }^{* *} p<0,01(2$-seitig) 
Das zweifaktorielle Modell C folgt der Unterscheidung in fachliche und überfachliche Kompetenzen bzw. Fähigkeiten (Blume et al. 2010). Hierbei laden die Fachkompetenzitems auf einem Faktor und die Methoden-, Sozial- und Selbstkompetenzitems auf dem zweiten Faktor. Das vierfaktorielle Modell D beinhaltet schließlich die in dieser Studie angenommenen vier latenten Faktoren Methoden-, Fach-, Sozial- und Selbstkompetenz (z. B. Erpenbeck und von Rosenstiel 2007; Kauffeld 2006; Sonntag und Schaper 2006).

Die Passung von Modell D ist als gut (RMSEA, CFI, SRMR) bzw. akzeptabel $\left(\chi^{2} / d f\right)$ einzuschätzen (siehe Tab. 3 für die Modellfit-Indizes, vgl. Schweizer 2010). In Modell D sind alle standardisierten Faktorladungen größer als der empfohlene Wert von 0,50 (Wirtz und Nachtigall 2012, S. 211). Die Reliabilitäten und Skaleninterkorrelationen sind in Tab. 3 angegeben. Wie in Hypothese 1 angenommen, kann eine finale vierfaktorielle Fragebogenstruktur erfolgreich identifiziert werden.

\subsection{Kriteriumsvalidität - Zusammenhang mit dem Weiterbildungserfolg}

Zur Prüfung der Kriteriumsvalidität wurden in Stichprobe 3 mehrere multiple Regressionsanalysen durchgeführt. Es wurden keine Kovariaten in den Analysen berücksichtigt, da keine signifikanten Interkorrelationen zwischen den Weiterbildungserfolgsmaßen (Note des/der Trainers/ Trainerin, Note des Trainings, wahrgenommene Anwendung in der Praxis) und potenziellen Kovariaten (Alter, Geschlecht, Organisationszugehörigkeit, Zeitraum zwischen Befragungsteilnahme und Teilnahme an der Weiterbildungsmaßnahme, Umfang der Weiterbildungsmaßnahme, Art des Inhalts der Weiterbildungsmaßnahme) vorliegen. Da die Interkorrelationen zwischen den vier manifesten Trainer/innenkompetenzdimensionen zwischen 0,62 und 0,83 lagen (siehe Tab. 3), wurde eine Multikollinearitätsdiagnose durchgeführt (z.B. Urban und Mayerl 2011). Die Varianzinflationsfaktoren (VIF-Werte zwischen 2,07 und 4,14) sowie die Toleranzen (Werte zwischen 0,24 und $0,48)$ über- bzw. unterschreiten jedoch nicht die entsprechenden Mindestgrenzwerte (VIF > 5 und Toleranz $<0,20$ für starke Multikollinearität laut Urban und Mayerl 2011, S. 232). Daher werden alle vier Kompetenzdimensionen in den Regressionsanalysen gleichzeitig berücksichtigt. Zur Überprüfung der gerichteten Hypothesen 2, 3 und 4 werden einseitige $p$-Werte angegeben.

Die vier Kompetenzbereiche klären gemeinsam $79 \%$ $(F(4,120)=111,50 ; p<0,01)$ an der globalen Zufriedenheit mit dem/der Trainer/Trainerin auf. Es zeigt sich ein signifikanter Zusammenhang zwischen allen vier Kompetenzbereichen und der globalen Bewertung des/der Trainers/ Trainerin (Methodenkompetenz $\beta=0,29 ; p<0,01$; Fachkompetenz $\beta=0,30 ; p<0,01$; Sozialkompetenz $\beta=0,19$; $p<0,05$; Selbstkompetenz $\beta=0,23 ; p<0,01)$. Je höher die vier Kompetenzbereiche des/der Trainers/Trainerin ausgeprägt sind, desto höher ist die Gesamtzufriedenheit mit dem/der Trainer/in ausgeprägt. Alle Teilhypothesen von H2 können auf Basis der Regressionsanalysen bestätigt werden.

Auf die globale Zufriedenheit mit der Weiterbildungsmaßnahme zeigen die vier Kompetenzbereiche eine gemeinsame Varianzaufklärung von $59 \%(F(4,120)=43,98$; $p<0,01)$. Drei der vier Kompetenzbereiche weisen einen signifikanten Zusammenhang mit der globalen Bewertung der Weiterbildungsmaßnahme auf (Methodenkompetenz: $\beta=0,32 ; p<0,01$; Fachkompetenz: $\beta=0,16 ; p<0,05$; Sozialkompetenz: $\beta=0,36 ; p<0,01)$. Einzig die Selbstkompetenz zeigt keinen signifikanten Zusammenhang $(\beta=0,03 ; p=0,41)$. Hypothese 3 kann also nur für drei (H3a: Methoden-, H3b: Fach- und H3c: Sozialkompetenz) der vier Kompetenzdimensionen bestätigt werden.

Auf den subjektiv wahrgenommenen Transfererfolg zeigen die vier Kompetenzbereiche eine gemeinsame Varianzaufklärung von $9 \%(F(4,120)=2,98 ; p<0,05)$. Es zeigt sich ein signifikanter Zusammenhang zwischen der Methodenkompetenz eines/einer Trainers/Trainerin und dem wahrgenommenen Transfererfolg der Teilnehmenden $(\beta=0,31 ; p<0,05)$, die anderen drei Kompetenzbereiche zeigen keinen signifikanten Zusammenhang mit dem Transfererfolg (Fachkompetenz: $\beta=-0,05 ; p=0,70$; Sozialkompetenz: $\beta=0,02 ; p=0,90$; Selbstkompetenz: $\beta=0,01$; $p=0,96)$. Somit kann nur Hypothese 4 a bestätigt werden.

\section{Diskussion}

Durch Zeitmangel und begrenzte Ressourcen sind praktikable und ökonomische Fragebögen für den Einsatz in Unternehmen unverzichtbar (Kauffeld 2016). Im vorliegenden Artikel wurde daher ein Kurzfragebogen zur zeiteffizienten Erfassung der Methoden-, Fach-, Sozial- und Selbstkompetenz von Trainern/Trainerinnen beruflicher Weiterbildungsmaßnahmen entwickelt. Konfirmatorische Faktorenanalysen legen eine vierfaktorielle Struktur (Methoden-, Fach-, Sozial- und Selbstkompetenz) nahe und geben Hinweise auf die faktorielle Validität des entwickelten Kurzfragebogens. Trotz substantieller Interkorrelationen zwischen den Kompetenzdimensionen zeigt eine Analyse von konkurrierenden Modellen (bspw. ein- oder zweifaktorielle Modelle) die Überlegenheit des vierfaktoriellen Modells. Die empirischen Daten liefern somit Hinweise auf die theoretisch postulierte Unterscheidung der vier Kompetenzdimensionen Methoden-, Fach-, Sozial- und Selbstkompetenz (Erpenbeck und von Rosenstiel 2007; Kauffeld 2006; Sonntag und Schaper 2006). Die hohen Interkorrelationen zwischen den Kompetenzdimensionen sind in guter Überein- 
stimmung mit theoretischen Überlegungen (Kauffeld 2006, S. 27) und zeigen, dass die vier Kompetenzdimensionen als nicht unabhängig voneinander zu betrachten sind.

Die Analysen zur Kriteriumsvalidität legen nahe, dass alle vier Kompetenzbereiche einen bedeutsamen Zusammenhang mit der Gesamtzufriedenheit mit dem/der Trainer/in aufweisen. Die drei Dimensionen Methoden-, Fachund Sozialkompetenz sind zudem relevant für die Zufriedenheit mit der Weiterbildungsmaßnahme. Die Ergebnisse können als Hinweise auf die konvergente Validität des Kurzfragebogens interpretiert werden. Die fehlende Relevanz der Selbstkompetenz eines/einer Trainers/Trainerin für die Zufriedenheit mit der Weiterbildungsmaßnahme kann darin begründet sein, dass diese Kompetenzdimension erst über andere Kompetenzdimensionen „zum Tragen“ kommt (Kauffeld 2006, S. 27). Die Selbstkompetenz (bspw. Offenheit für Veränderungen) könnte sich folglich über einen flexiblen und kreativen Umgang mit Methoden zur Vermittlung der Weiterbildungsinhalte (d.h. eine hohe Methodenkompetenz) auf die Zufriedenheit mit der Maßnahme auswirken (vgl. Kauffeld 2006). Allerdings können auch methodische Gründe für den nicht-signifikanten Einfluss der Selbstkompetenz ursächlich sein: So steigen mit höheren Interkorrelationen auch die Standardfehler, was eine Schätzung der Regressionsgewichte unzuverlässiger macht (Urban und Mayerl 2011). Die berechneten VIF- und Toleranzwerte zeigen jedoch, dass die Mindestgrenzwerte für starke Multikollinearität nicht über- bzw. unterschritten werden.

Mit dem wahrgenommenen Transfererfolg der Teilnehmenden zeigt sich ausschließlich für die Methodenkompetenz eines/einer Trainers/Trainerin ein signifikanter Zusammenhang, was als erster Hinweis auf die diskriminante Validität des Kurzfragebogens interpretiert werden kann. Dies ist in guter Übereinstimmung mit bisheriger Forschung, die bspw. zeigte, dass eine hohe Übereinstimmung zwischen den vom Trainer oder der Trainerin vermittelten Inhalten und dem Arbeitsalltag transferbegünstigend wirkt (z.B. Lim 2000); ein Aspekt der eher der Methodenkompetenz eines/einer Trainers/Trainerin zuzuordnen ist.

Kritisch anzumerken ist, dass in den vorliegenden Online-Studien Gelegenheitsstichproben von Berufstätigen erhoben wurden. Durch eine randomisierte Auswahl von Probanden/Probandinnen könnten mögliche Verzerrungen der Ergebnisse durch Selbstselektion der Teilnehmenden ausgeschlossen und die Repräsentativität der Stichproben sichergestellt werden (z. B. Sachs und Hedderich 2006). Darüber hinaus könnte eine gezielte Auswahl unterschiedlicher Arten beruflicher Weiterbildungsmaßnahmen in zukünftigen Studien einen systematischen Vergleich der Kompetenzdimensionen erlauben. Allerdings war es mit dem gewählten Forschungsdesign möglich, zu zeigen, dass der entwickelte Fragebogen über verschiedene Branchen und Weiterbildungsinhalte hinweg anwendbar ist.
In allen drei Studien wurden die Daten retrospektiv zu einem Messzeitpunkt erfasst. In längsschnittlichen Studien sollten die hier vorgestellten psychometrischen Ergebnisse daher weiter abgesichert werden. Durch die Messung der Trainer/innenkompetenz direkt nach der Weiterbildungsmaßnahme könnten insbesondere Erinnerungseffekte bezüglich spezifischer Methoden und Vorgehensweisen der Trainer/innen vermieden werden. Fragen zum Transfer hingegen sollten mit ausreichend Abstand zur Weiterbildungsmaßnahme gestellt werden, damit nicht nur ein kurzfristiger Effekt, sondern ein langfristiger Transfer betrachtet werden kann. Optimalerweise sollte die Messung des Transfers auch zu unterschiedlichen Zeitpunkten erfolgen, da diese abhängig ist von der seit der Weiterbildungsmaßnahme vergangenen Zeit (Blume et al. 2010). Die Querschnittliche Betrachtung in den vorliegenden Stichproben könnte auch eine Erklärung für nicht gefundene Effekte sein. Durch die retrospektive Erfassung war es jedoch möglich eine heterogene Stichprobe mit einer geringen Anzahl fehlender Werte zu erheben und somit erste wertvolle Einblick zu erhalten. Darüber hinaus sollten in zukünftigen Studien weitere Merkmale der Weiterbildungsmaßnahme, des Arbeitsumfelds und der Teilnehmenden (z.B. Aguinis und Kraiger 2009) als Einflussfaktoren auf den Weiterbildungserfolg berücksichtigt werden, um den Beitrag des/der Trainers/Trainerin im gesamten Transfersystem abschätzen zu können. So zeigt die Fallstudie von Donovan (2017) beispielsweise, dass die organizationale Unterstützung der entscheidende Faktor für eine Verhaltensänderung ist - nicht der/die Trainer/in. Dies könnte auch eine Erklärung für die geringen Zusammenhänge der Trainer/ innenkompetenz mit dem wahrgenommenen Transfer sein. Eine Berücksichtigung diverser potenzieller Einflussfaktoren kann somit Aufschluss über entscheidende Stellgrößen für den Trainigstransfer liefern und somit die Grundlage geben, um die Relevanz des/der Trainers/Trainerin in diesem Kontext besser zu beurteilen.

Im vorliegenden Artikel wurden die Trainer/innenkompetenzen mittels Fremdeinschätzung durch die Teilnehmenden und die Maße des Weiterbildungserfolgs mittels Selbsteinschätzung durch die Teilnehmenden erhoben. Durch die Einschätzung von nur einer Person, kann es zu einer Überschätzung der Zusammenhänge zwischen Trainer/innenkompetenzen und wahrgenommenem Transfer gekommen sein (Blume et al. 2010). Zudem ist auch fraglich, inwiefern der subjektiv wahrgenommene Transfer die Realität abbildet. In künftigen Validierungsstudien sollten zur Einschätzung des Weiterbildungserfolgs daher neben umfassenderen Selbsteinschätzungsmaßen ebenfalls objektive, externe Maße (für Beispiele, siehe Aguinis und Kraiger 2009) eingesetzt werden. Auch wenn die hier verwendeten Maße des Weiterbildungserfolgs bereits in früheren Studien erfolgreich angewendet wurden (z.B. Grohmann 
und Kauffeld 2013; Staufenbiel 2000), kann erst durch objektive Maße eine finale Aussage über die Zusammenhänge gemacht werden. Aufgrund der Fremdeinschätzung der Trainer/innenkompetenz durch Laien ohne pädagogischen Hintergrund ist weiterhin zu beachten, dass lediglich eine Aussage darüber getroffen werden kann, ob die Teilnehmenden die Trainingsmethoden subjektiv als passend empfunden haben - nicht jedoch ob aus pädagogischer Sich die richtige Methode verwendet wurde.

Der entwickelte Kurzfragebogen ist in einem breiten Spektrum beruflicher Weiterbildungsmaßnahem einsetzbar. Wird eine umfassende und zeitintensive Einschätzung von Trainer/innenkompetenzen angestrebt, dann sollte der Kurzfragebogen jedoch in zukünftigen Studien durch domänenspezifische Instrumente ergänzt werden (vgl. Sgier und Lattke 2012). Darüber hinaus könnte bspw. auch eine vertiefende externe Evaluation durch eine/n autorisierte/n, externe/n Evaluator/in, wie sie im Projekt Validpack vorgesehen ist (Strauch 2008), mit dem Kurzfragebogen kombiniert werden.

Insgesamt lässt sich festhalten, dass mit dem Kurzfragebogen zur Erfassung von Trainer/innenkompetenzen in beruflichen Weiterbildungsmaßnahmen die vier Kompetenzbereiche eines/einer Trainers/Trainerin (Methoden-, Fach-, Sozial- und Selbstkompetenz) beruflicher Weiterbildungsmaßnahmen zeitökonomisch erfasst werden. Der entwickelte Kurzfragebogen kann somit zur kontinuierlichen Evaluation in der Praxis beruflicher Weiterbildungsmaßnahmen eingesetzt werden. Auch wenn sich für eine umfassende Reflexion der eigenen Kompetenzen andere Instrumente besser eigenen, wie bspw. das PortfolioPlus aus dem GRETA Projekt (Bosche et al. 2018), kann unser Kurzfragebogen die Reflexion der eigenen Trainer/innenkompetenzen unterstützen. So können bspw. Berufsanfänger/innen diesen Fragebogen als Basis nutzen, um ihre Kompetenzen kontinuierlich weiterzuentwickeln. Während umfassende Inventare viel Zeit in Anspruch nehmen und daher in der Regel nicht wiederholt eingesetzt werden, ermöglicht der Kurzfragebogen eine kontinuierliche Begleitung und Weiterentwicklung. Bei Bedarf können dann vertiefende Analyseinstrumente eingesetzt werden. Auch in Ausbildungen für Trainer/innen kann das Instrument somit unterstützend eingesetzt werden. Unternehmen und Weiterbildungsanbieter können zudem einen besonderen Fokus auf die Entwicklung der Methodenkompetenz von eingesetzten Trainern/ Trainerinnen setzen, um so den Transfererfolg von Weiterbildungsmaßnahmen zu erhöhen.

Danksagung Wir danken Philipp Schaper für die Unterstützung bei der Datenerhebung für Studie 1 und den fachlichen Austausch bei der Konstruktion des Kurzfragebogens. Wiebke Brune danken wir für die Unterstützung bei der Datenerhebung für Studie 2. Erste Voranalysen zu Studie 1 wurden 2010 beim 47. Kongress der Deutschen Gesellschaft für Psychologie in Bremen präsentiert.
Funding Open Access funding enabled and organized by Projekt DEAL.

Open Access Dieser Artikel wird unter der Creative Commons Namensnennung 4.0 International Lizenz veröffentlicht, welche die Nutzung, Vervielfältigung, Bearbeitung, Verbreitung und Wiedergabe in jeglichem Medium und Format erlaubt, sofern Sie den/die ursprünglichen Autor(en) und die Quelle ordnungsgemäß nennen, einen Link zur Creative Commons Lizenz beifügen und angeben, ob Änderungen vorgenommen wurden.

Die in diesem Artikel enthaltenen Bilder und sonstiges Drittmaterial unterliegen ebenfalls der genannten Creative Commons Lizenz, sofern sich aus der Abbildungslegende nichts anderes ergibt. Sofern das betreffende Material nicht unter der genannten Creative Commons Lizenz steht und die betreffende Handlung nicht nach gesetzlichen Vorschriften erlaubt ist, ist für die oben aufgeführten Weiterverwendungen des Materials die Einwilligung des jeweiligen Rechteinhabers einzuholen.

Weitere Details zur Lizenz entnehmen Sie bitte der Lizenzinformation auf http://creativecommons.org/licenses/by/4.0/deed.de.

\section{Literatur}

Aguinis, H., \& Kraiger, K. (2009). Benefits of training and development for individuals and teams, organizations, and society. Annual Review of Psychology, 60, 451-474.

Arghode, V., \& Wang, J. (2016). Exploring trainers' engaging instructional practices: a collective case study. European Journal of Training and Development, 40(2), 111-127.

Baldwin, T.T., Ford, K. J., \& Blume, B.D. (2017). The state of transfer of training research: moving toward more consumer-centric inquiry. Human Resource Development Quarterly, 28, 17-28.

Beicht, U., Krekel, E. M., \& Walden, G. (2006). Berufliche Weiterbildung: Welche Kosten und welchen Nutzen haben die Teilnehmenden? Bielefeld: Bertelsmann.

Bell, S. T., Towler, A. J., \& Fisher, D. M. (2011). A multilevel examination of the influence of trainee-trainer gender dissimilarity and trainee-classroom gender composition dissimilarity on trainee knowledge acquisition. Human Resource Development Quarter$l y, 22,343-372$.

Bentler, P. M., \& Chou, C.-P. (1987). Practical issues in structural modeling. Sociological Methods \& Research, 16, 78-117.

Blau, G., Gibson, G., Bentley, M., \& Chapman, S. (2012). Testing the impact of job-related variables on a utility judgment training criterion beyond background and affective reaction variables. International Journal of Training and Development, 16, 54-66.

Blume, B. D., Ford, J. K., Baldwin, T. T., \& Huang, J. L. (2010). Transfer of training: a meta-analytic review. Journal of Management, $36,1065-1105$.

Bonnes, C., Wißhak, S., \& Hochholdinger, S. (2019). Towards a model of professional competence of workplace trainers. In M. Pilz, K. Breuing \& S. Schumann (Hrsg.), Berufsbildung zwischen Tradition und Moderne. Internationale Berufsbildungsforschung. Wiesbaden: Springer VS.

Bosche, B., Schneider, M., \& Strauch, A. (2018). Lehrkompetenzen in der Erwachsenen- und Weiterbildung: Bilanzierung und Möglichkeiten der Anerkennung mit dem Instrument PortfolioPlus. Berufsbildung in Wissenschaft und Praxis, 47(1), 47-50.

Bühner, M. (2011). Einführung in die Test- und Fragebogenkonstruktion (3. Aufl.). München: Pearson Studium.

Burke, L. A., \& Hutchins, H. M. (2008). A study of best practices in training transfer and proposed model of transfer. Human Resource Development Quarterly, 19, 107-128.

Donovan, P. (2017). Finding the critical few: The hot buttons of training transfer at ICON-A case study in evaluation and learn- 
ing transfer at a global CRO (Clinical Research Organization). In S. Frasard \& F. C. Prasuhn (Hrsg.), Training initiatives and strategies for the modern workforce (S. 131-161). Hershey: IGI Global.

Donovan, P., \& Darcy, D.P. (2011). Learning transfer: the views of practitioners in ireland. International Journal of Training and Development, 15, 121-139.

Erpenbeck, J., \& v. Rosenstiel, L. (Hrsg.). (2007). Handbuch Kompetenzmessung: Erkennen, verstehen und bewerten von Kompetenzen in der betrieblichen, pädagogischen und psychologischen Praxis (2. Aufl.). Stuttgart: Schäffer-Poeschel.

Fisseni, H.-J. (2004). Lehrbuch der psychologischen Diagnostik (3. Aufl.). Göttingen: Hogrefe.

Flanagan, J.C. (1954). The critical incident technique. Psychological Bulletin, 51, 327-358.

Garrick, J., \& McDonald, R. (1992). Competency-based standards for industry trainers: a critical comparison of international models. Studies in Continuing Education, 14, 166-186.

Gauld, D., \& Miller, P. (2004). The qualifications and competencies held by effective workplace trainers. Journal of European Industrial Training, 28, 8-22.

Graf, N. (2011). Sozialkompetenzen von Trainern in der betrieblichen Weiterbildung. Hamburg: Dr. Kovač.

Grohmann, A., \& Kauffeld, S. (2013). Evaluating training programs: development and correlates of the Questionnaire for Professional Training Evaluation. International Journal of Training and Development, 17, 135-155.

Grohmann, A., Beller, J., \& Kauffeld, S. (2014). Exploring the critical role of motivation to transfer in the training transfer process. International Journal of Training and Development, 18, 84-103.

Harris, R., Simons, M., \& Bone, J. (2000). More than meets the eye? Rethinking the role of workplace trainer. Adelaide: National Centre for Vocational Education Research.

Harris, T. B., Chung, W., Frye, C.L., \& Chiaburu, D. S. (2014). Satisfaction guaranteed? Enhanced impact of trainer competence for autonomous trainees. Industrial and Commercial Training, 46(5), 270-277.

Hochholdinger, S., \& Keller, I. (2015). Welche Zusammenhänge bestehen zwischen den affektiven Reaktionen von Trainingsteilnehmenden, der Lehrorientierung von Weiterbildungspersonal und Weiterbildungsinhalten? Zeitschrift für Erziehungswissenschaft, 18(S1), 123-144.

Hölbling, G. (2007). Handlungshilfen für Bildungsberater: Qualitätssicherung betrieblicher Weiterbildung. Band 23, Schriftenreihe des Forschungsinstituts Betriebliche Bildung gGmbH. Bielefeld: Bertelsmann.

Holton III, E. F., Bates, R., \& Ruona, W.E. A. (2000). Development of a generalized learning transfer system inventory. Human Resource Development Quarterly, 11, 333-360.

Hughes, C., Toohey, S., \& Hatherly, S. (1992). Developing learningcentered trainers and tutors. Studies in Continuing Education, 14, $14-27$.

Kauffeld, S. (2006). Kompetenzen messen, bewerten, entwickeln. Stuttgart: Schäffer-Poeschel.

Kauffeld, S. (2016). Nachhaltige Personalentwicklung und Weiterbildung. Betriebliche Seminare und Trainings entwickeln, Erfolge messen, Transfer sichern (2. Aufl.). Berlin: Springer.

Kauffeld, S., \& Henschel, A. (2010). Das Kompetenz-Reflexions-Inventar (KRI). In W. Sarges \& H. Wottawa (Hrsg.), Handbuch wirtschaftspsychologischer Testverfahren (2. Aufl. S. 201-207). Lengerich: Pabst.

Kauffeld, S., \& Paulsen, H.F.K. (2018). Kompetenzmanagement in Unternehmen: Kompetenzen beschreiben, messen, entwickeln und nutzen. Stuttgart: Kohlhammer.

Kirkpatrick, D.L., \& Kirkpatrick, J.D. (2006). Evaluating training programs: the four levels (3. Aufl.). San Francisco: Berrett-Koehler.

Kline, R. B. (2005). Principles and practice of structural equation modeling (2. Aufl.). New York: Guilford.
Lim, D. H. (2000). Training design factors influencing transfer of training to the workplace within an international context. Journal of Vocational Education and Training, 52, 243-258.

Mamaqi, X., Miguel, J., \& Olave, P. (2011). Evaluation of the importance of professional competences: the case of Spanish trainers. On the Horizon, 19(3), 174-187.

Marx, C., Goeze, A., Voss, T., Hoehne, V., Klotz, V. K., \& Schrader, J. (2017). Pädagogisch-psychologisches Wissen von Lehrkräften aus Schule und Erwachsenenbildung: Entwicklung und Erprobung eines Testinstruments. Zeitschrift für Erziehungswissenschaft, 20(S1), 165-200.

Mayring, P. (2010). Qualitative Inhaltsanalyse: Grundlagen und Techniken (11. Aufl.). Weinheim: Beltz.

Morgan, R. B., \& Casper, W. J. (2000). Examining the factor structure of participant reactions to training: A multidimensional approach. Human Resource Development Quarterly, 11, 301-317.

Muthén, L. K., \& Muthén, B. O. (2010). Mplus user's guide (6. Aufl.). Los Angeles: Muthén \& Muthén.

Nor, D.A.M., Saadon, M.S.I., Kowang, T.O., Khan, M.N.A.A., \& Fei, G.C. (2017). A critical review on the dimensions of transfer of training. Advanced Science Letters, 23(9), 8566-8568.

Olson, S. J. (1994). Competencies of two-year college technical instructors and technical trainers: similarities and differences. Journal of Industrial Teacher Education, 32(1), 65-85. http://scholar. lib.vt.edu/ejournals/JITE/. Gesehen 27. September 2012.

Oser, F., Salzmann, P., \& Heinzer, S. (2009). Measuring the competence-quality of vocational teachers: an advocatory approach. Empirical Research in Vocational Education and Training, 1, 65-83.

Russ-Eft, D.F., Dickison, P., \& Levine, R. (2010). Taking the pulse of training transfer: Instructor quality and EMT certification examination results. Human Resource Development Quarterly, 21(3), 291-306.

Sachs, L., \& Hedderich, J. (2006). Angewandte Statistik (12. Aufl.). Berlin: Springer.

Schwaab, M.-O. (2002). Strukturierte Auswahl externer Trainer: Eine empirische Studie zum Bildungscontrolling. München: Hampp.

Schweizer, K. (2010). Some guidelines concerning the modeling of traits and abilities in test construction. European Journal of Psychological Assessment, 26, 1-2.

Sgier, I. (2012). Kernkompetenzen für die Erwachsenenbildung aus Schweizer und aus internationaler Sicht: Resultate aus dem Forschungsprojekt QF2TEACH. In I. Sgier \& S. Lattke (Hrsg.), Professionalisierungsstrategien der Erwachsenenbildung in Europa: Entwicklungen und Ergebnisse aus Forschungsprojekten (S. 127-144). Bielefeld: Bertelsmann.

Sgier, I., \& Lattke, S. (Hrsg.). (2012). Professionalisierungsstrategien der Erwachsenenbildung in Europa: Entwicklungen und Ergebnisse aus Forschungsprojekten. Bielefeld: Bertelsmann.

Sitzmann, T., \& Weinhardt, J. M. (2019). Approaching evaluation from a multilevel perspective: a comprehensive analysis of the indicators of training effectiveness. Human Resource Management Review, 29(2), 253-269.

Sonntag, K., \& Schäfer-Rauser, U. (1993). Selbsteinschätzung beruflicher Kompetenzen bei der Evaluation von Bildungsmaßnahmen. Zeitschrift für Arbeits- und Organisationspsychologie, 37, 163-169.

Sonntag, K., \& Schaper, N. (2006). Förderung beruflicher Handlungskompetenz. In K. Sonntag (Hrsg.), Personalentwicklung in Organisationen (3. Aufl. S. 270-311). Göttingen: Hogrefe.

Staufenbiel, T. (2000). Fragebogen zur Evaluation von universitären Lehrveranstaltungen durch Studierende und Lehrende. Diagnostica, 46, 169-181.

Stoughton, J. W., Gissel, A., Clark, A. P., \& Whelan, T. J. (2011). Measurement invariance in training evaluation: old question, new context. Computers in Human Behavior, 27, 2005-2010.

Strauch, A. (2008). Validation of adult educators ' competencies: the VINEPAC project. Lifelong Learning in Europe (Lline), 13(3), 181-187. 
Towler, A. (2009). Effects of trainer expressiveness, seductive details, and trainee goal orientation on training outcomes. Human Resource Development Quarterly, 20, 65-84.

Urban, D., \& Mayerl, J. (2011). Regressionsanalyse: Theorie, Technik und Anwendung (4. Aufl.). Wiesbaden: VS.

Wang, G. G., \& Wilcox, D. (2006). Training evaluation: knowing more than is practiced. Advances in Developing Human Resources, 8 , $528-539$.

Wick, A. (2009). Kontextabhängigkeit der Auswahl und Brauchbarkeit von Messverfahren zur Diagnose beruflicher Kompetenzen. report - Zeitschrift für Weiterbildungsforschung, 3, 24-34.

Wirtz, M., \& Nachtigall, C. (2012). Deskriptive Statistik: Statistische Methoden für Psychologen Teil 1 (6. Aufl.). Weinheim: Juventa.

Wißhak, S., \& Hochholdinger, S. (2018). Trainers' knowledge and skills from the perspective of trainers, trainees and human resource development practitioners. International Journal of Training Research, 16(3), 218-231.

Wißhak, S., Bonnes, C., Keller, I., Barth, D., \& Hochholdinger, S. (2020). Qualifikationen von Lehrenden in der beruflich-betrieblichen Weiterbildung. Zeitschrift für Bildungsforschung, 10(1), $103-123$.

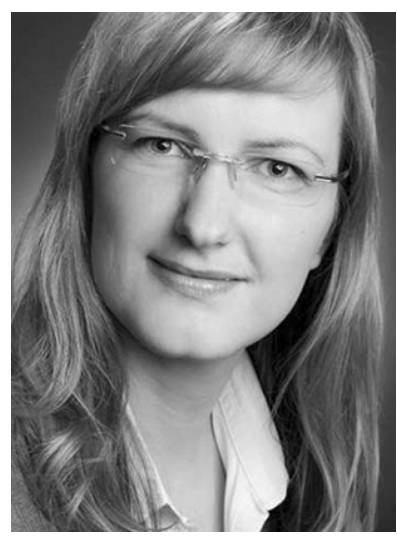

Dipl-Psych. A. Grohmann arbeitete von 2008 bis 2013 als wissenschaftliche Mitarbeiterin am Lehrstuhl für Arbeits-, Organisationsund Sozialpsychologie an der Technischen Universität Braunschweig. Sie war in dieser Zeit regelmäßig als Trainerin tätig. Auch in ihrer Forschung befasste sie sich mit dem Thema Training und Transfer. Weitere Forschungsgebiete waren die Evaluation von Weiterbildungsmaßnahmen sowie die Test- und Fragebogenkonstruktion.

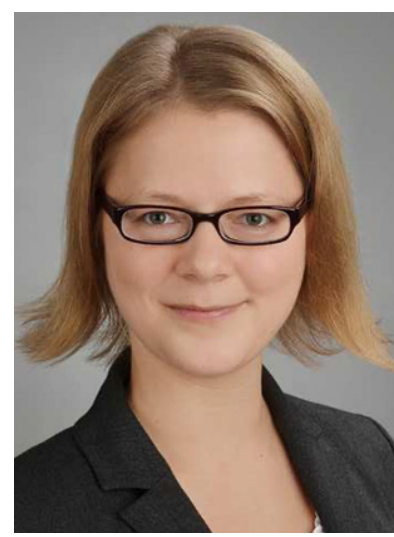

Dr. E.-M. Schulte ist seit 2009 wissenschaftliche Mitarbeiterin am Lehrstuhl für Arbeits-, Organisations- und Sozialpsychologie an der Technischen Universität Braunschweig. Ihre Forschungsschwerpunkte umfassen Gesunde Arbeit (u.a. Resilienz, Anforderungen \& Ressourcen, Verhaltensprävention), Gesunde Führung (u. a. Förderung gesunder Führung, LMX, Teamresilienz, Verhältnisprävention, Gestaltung von Meetings), Coaching (u. a. Tools im Coaching, Selbstwirksamkeit, Karriereentwicklung) sowie Training \& Transfer (u. a. Evaluation, entwicklungsorientierte Begleitung). Seit 2016 ist sie zudem als Senior Consultant bei der 4A-SIDE GmbH tätig (Schwerpunkte Training und Coaching).

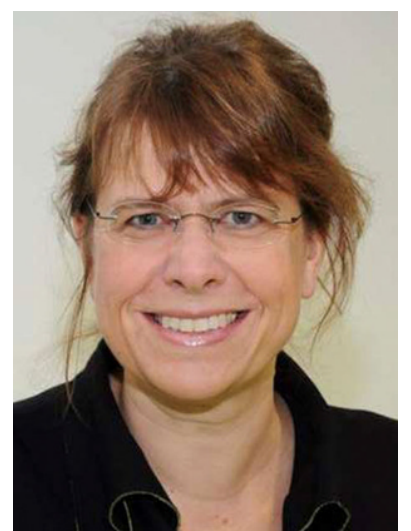

Prof. Dr. S. Kauffeld ist Inhaberin des Lehrstuhls für Arbeits-, Organisations- und Sozialpsychologie der Technischen Universität Braunschweig. In ihrer Forschungstätigkeit setzt sie sich mit den Themen Kompetenz, Team, Beratung und Führung auseinander. Um ihre Konzepte der Praxis zugänglich $\mathrm{zu}$ machen, hat sie 2008 unter Beteiligung der TU Braunschweig die 4ASIDE GmbH gegründet. 